\title{
Noch immer auf der Suche nach dem heiligen Gral: Wie generisch oder fachspezifisch sind Dimensionen der Unterrichtsqualität?
}

\author{
Anna-Katharina Praetorius • Cornelia Gräsel
}

Eingegangen: 20. April 2021 / Überarbeitet: 14. Mai 2021 / Angenommen: 19. Mai 2021 / Online publiziert: 23. Juni 2021

(C) Der/die Autor(en) 2021

Zusammenfassung Im vorliegenden Beitrag wird der Versuch unternommen, zentrale Aspekte der elf Positionsbeiträge des vorliegenden Themenheftes „Unterrichtsqualität - fachspezifisch oder generisch? Ein Update zu Perspektiven aus der Fachdidaktik und der allgemeinen Lehr-Lernforschung“ (Heft 2, 2021) im Hinblick auf die Frage nach der Fachspezifität von Dimensionen der Unterrichtsqualität zusammenzufassen und zu diskutieren. Als Grundlage für den Vergleich wird ein Syntheseframework verwendet, zu dem auch die Autor*innen des Themenheftes Stellung genommen haben. Es zeigt sich ein großes Ausmaß an Gemeinsamkeiten zwischen unterschiedlichen Fächern, darüber hinaus auch etliche fachspezifische Ergänzungsvorschläge (u.a. in Bezug auf kognitive Aktivierung und sozio-emotionale Unterstützung). Abschließend werden erste Ideen für eine zukünftige Weiterentwicklung der Frage nach der Fachspezifität im Allgemeinen sowie des Syntheseframeworks im Speziellen vorgeschlagen, die sich auf die Ziele von Unterricht, die Berücksichtigung von Lernprozessen sowie die Verbesserung von Unterrichtsqualität beziehen.

Schlüsselwörter Fachspezifität · Unterrichtsqualität · Kognitive Aktivierung · Unterstützung des Übens

Die Autoren haben zu gleichen Teilen zum Manuskript beigetragen.

Literaturangaben die mit einem \# markiert sind, sind Teil des vorliegenden Themenhefts.

Anna-Katharina Praetorius $(\triangle)$

Professur für pädagogisch-psychologische Lehr-Lernforschung und Didaktik, Institut für Erziehungswissenschaft, Universität Zürich, Zürich, Schweiz

E-Mail: anna.praetorius@ife.uzh.ch

Cornelia Gräsel $(\bowtie)$

Institut für Bildungsforschung in der School of Education, Bergische Universität Wuppertal,

Wuppertal, Deutschland

E-Mail: graesel@uni-wuppertal.de 


\title{
Still searching for the holy grail: How generic or subject-specific are dimensions of teaching quality?
}

\begin{abstract}
This paper is an attempt to summarize key points about the subject specificity of dimensions of teaching quality made in the eleven papers in this special issue, "Teaching quality: Updating different perspectives on general and subjectspecific research on learning and instruction" (issue 2, 2021). For the comparison, a synthesis framework was used that was also the basis for the other papers in this issue. It resulted in the identification of many commonalities across all subjects. The synthesis also revealed that some dimensions of teaching quality need subject specific adaptations (e.g., cognitive activation and socio-emotional support). We provide some initial ideas for further research on the subject specificity of teaching quality in general and the development of the synthesis framework in particular. The ideas address teaching goals, learning processes, and the overall improvement of teaching quality.
\end{abstract}

Keywords Subject specificity · Teaching quality $\cdot$ Instructional quality $\cdot$ Cognitive activation $\cdot$ Support in practice

\section{Einleitung}

Dieses Themenheft begibt sich erneut auf die Suche nach dem „Heiligen Gral“ der Unterrichtsforschung (vgl. Neumann 2018), also derjenigen Dimensionen, die qualitätsvollen Unterricht in unterschiedlichen Fächern auszeichnen. Die Gralsritter*innen, die sich zur Beantwortung dieser Frage versammeln, weisen zunächst hohes Durchhaltevermögen auf: Seit nunmehr gut 50 Jahren wird in der empirischen Unterrichtsforschung diskutiert, was Unterrichtsqualität konstituiert und durch welche Dimensionen sie beschrieben werden kann. In der allgemeinen Unterrichtsforschung wird dabei traditionell vorrangig effektiver Unterricht in den Blick genommen (z. B. Klieme 2019; Seidel und Shavelson 2007), also jene Dimensionen, die damit einhergehen, dass die jeweils intendierten Lernziele erreicht werden - wobei der Schwerpunkt bislang auf Wirkungen in Hinblick auf den fachlichen Wissensoder Kompetenzerwerb liegt. Der Anspruch der allgemeinen Unterrichtsforschung bestand und besteht in der Formulierung generischer Dimensionen (z. B. Lindmeier und Heinze 2020; Reusser und Pauli 2021\#1), aber die überwiegende Zahl der Forschungsarbeiten wurde im Mathematikunterricht und - in geringerem Umfang - im naturwissenschaftlichen Unterricht durchgeführt (siehe Kunter und Ewald 2016). In den letzten Jahren entstanden verstärkt auch in anderen Fächern empirische Arbeiten, weil sich Fachdidaktiker*innen neben der Frage nach „,gutem“ Unterricht, der bestimmten fachlichen oder fachdidaktischen Prinzipien entspricht, vermehrt mit

\footnotetext{
1 Wir verweisen in unserem Beitrag häufig auf die im selben Themenheft erschienenen Aufsätze. Damit Leser*innen diese Verweise auf einen Blick nachvollziehen und von anderweitigen Publikationen im selben Jahr unterscheiden können, haben wir die Beiträge des vorliegenden Themenheftes mit einem \#-Zeichen gekennzeichnet.
} 
effektivem Unterricht befassen (von Reusser und Pauli 2021\# als fachdidaktische Wende bezeichnet). Die Ritter*innenrunde wurde damit nicht nur größer, sondern auch in ihrer Herkunft und Perspektivierung heterogener. Bislang wurde jedoch nur vereinzelt in den Blick genommen (z.B. Hellermann et al. 2021; Rothgangel et al. 2021; Schilcher et al. 2021), in welchem Ausmaß sich die Sichtweisen substanziell unterscheiden und in welchem Ausmaß Gemeinsamkeiten zwischen Fächern identifiziert werden können. Dies wird daher im vorliegenden Themenheft mit Fokus auf Gemeinsamkeiten und Unterschiede hinsichtlich zentraler Dimensionen der Unterrichtsqualität explizit fokussiert.

\section{Zur Idee des Heftes und des vorliegenden Beitrags}

Das Themenheft 2/2021 „Unterrichtsqualität - fachspezifisch oder generisch? Ein Update zu Perspektiven aus der Fachdidaktik und der allgemeinen Lehr-Lernforschung" schließt an die angestoßene Diskussion in Heft 3/2020 der Unterrichtswissenschaft an, in dem der aktuelle Forschungsstand zu Unterrichtsqualität in den Naturwissenschaften, dem Sport und der Geschichte präsentiert wurde. Darin wurde exemplarisch erarbeitet, welche Gemeinsamkeiten und Spezifika von Dimensionen der Unterrichtsqualität sich in diesen Fächern finden. In Fortführung dieser Auseinandersetzung haben wir für das vorliegende Themenheft namhafte Expert*innen diverser Fächer um ein kurzes Positionspapier gebeten. Sie sollten zunächst einen Überblick über die Diskussionen in ihren jeweiligen Disziplinen zur Frage geben, inwiefern Dimensionen der Unterrichtsqualität fachspezifisch oder fachübergreifend gesehen werden. Daran anknüpfend sollten sie ihre eigene Position zu dieser Frage darstellen. Konkret wurden zunächst erneut Vertreter*innen der bereits im Thementeil 3/2020 vertretenen Fächer/Fachgruppen eingeladen (siehe Lehr-Lernforschung, Reusser und Pauli 2021\#; Sport, Richartz und Kohake 2021\#; Geschichte, Trautwein et al. 2021\#; Biologie, Neuhaus 2021\#). Dadurch sollte zum einen die Kontinuität zwischen den beiden Thementeilen sichergestellt werden und zum anderen die Subjektivität verringert werden, die mit einer Diskussion des Ausmaßes an Generik/ Fachspezifität von Unterrichtsqualität einhergeht. Darüber hinaus tragen zur Erweiterung des Bildes zu diesem Heft Expert*innen weiterer Fächer bei (siehe Deutsch, Wiprächtiger-Geppert et al. 2021\#; Englisch, Wilden 2021\#; Kunst, Rakoczy et al. 2021\#; Musik, Kranefeld 2021\#; Religion, Rothgangel 2021\#; Mathematik, Dreher und Leuders 2021\#). Für einige Beiträge haben sich Forschende der allgemeinen Lehr-Lernforschung und einer Fachdidaktik zusammengetan, um mit beiden Brillen den Forschungsstand im jeweiligen Fach zu reflektieren (Kunst; Geschichte). Ein weiterer Beitrag schließlich vereint verschiedene Perspektiven mit dem Fokus, für die Erforschung von Unterrichtsqualität neben der Fachspezifität auch alternative Ideen - z. B. eine Orientierung an Inhalten - aufzuwerfen (Seidel et al.\#).

Die Reihenfolge der Heftbeiträge möchten wir folgendermaßen begründen: Den fachdidaktischen Beiträgen vorangestellt ist ein Beitrag, der die Entwicklung der Diskussion um Qualitätsdimensionen unter Bezug auf verschiedene theoretische Ansätze darstellt. Nachfolgend werden die Aufsätze aus den verschiedenen Fachdidaktiken geordnet nach Fächergruppen präsentiert; wir beginnen mit den geis- 
teswissenschaftlichen und musisch-ästhetischen Fächern, die bisher vergleichsweise weniger thematisiert wurden, gefolgt von mathematisch-naturwissenschaftlichen Fächern. Den Abschluss bildet der Aufsatz, in dem allgemeine und fachdidaktische Perspektiven kombiniert und ein Weg für weitere Entwicklungen der Unterrichtsqualitätsforschung gewiesen wird.

Als gemeinsamen Bezugspunkt aller Beiträge haben wir die Autor*innen gebeten, auf das erweiterte Syntheseframework ${ }^{2}$ zu Dimensionen der Unterrichtsqualität Bezug zu nehmen, das im vorangegangenen Thementeil mit allen Beteiligten erarbeitet wurde (und seinerseits aufbaut auf Praetorius und Charalambous 2018). Das erweiterte Syntheseframework umfasst sieben Dimensionen (siehe Tab. 1), die sich am Lernprozess der Schüler*innen orientieren. Die ersten vier Dimensionen werden dabei als direkt auf einzelne Schritte des Lernprozesses ausgerichtet konzeptualisiert, während angenommen wird, dass die drei anderen Dimensionen alle Schritte des Lernprozesses indirekt beeinflussen.

Die Wahl des Syntheseframeworks als Vergleichsgrundlage für das vorliegende Themenheft ist begründungspflichtig, insbesondere weil es sich hierbei um einen erst kürzlich entwickelten, konzeptuellen Ansatz handelt, der noch nicht als im Feld etabliert und empirisch überprüft gelten kann: Zunächst einmal bietet das Syntheseframework den Vorteil, dass es auf Basis einer Analyse über zwölf im internationalen Raum genutzte beobachtungsbasierte Frameworks entwickelt wurde. Dabei wurden erstens generische, zweitens mathematikspezifische und drittens hybride (also kombiniert generisch und mathematikspezifische) Frameworks aufgenommen. Das Syntheseframework wurde anschließend hinsichtlich seiner Passung für weitere Fächer überprüft und ergänzt (für die Notwendigkeit einer solchen Überprüfung, siehe Rothgangel 2021\#). Das erweiterte Syntheseframework bietet daher eine aktuelle Übersicht über bedeutsame generische und fachspezifische Dimensionen von Unterrichtsqualität über Fächer hinweg (siehe Praetorius et al. 2020a). Damit weicht es in seiner Zielsetzung von anderen Frameworks ab. Es wurde nicht mit der vorrangigen Intention formuliert, möglichst sparsam jene Dimensionen zu identifizieren, die über viele Studien und Kontexte hinweg modelliert werden können. Dafür ist das vielfach empirisch in den Blick genommene Modell der drei Basisdimensionen (Klieme 2019; Praetorius et al. 2018), wie von Trautwein et al. (2021\#) hervorgehoben, auch in unseren Augen besser geeignet. Vielmehr besteht ein zentrales Ziel darin, eine integrative Systematisierung generischer und fachspezifischer Dimensionen von Unterrichtsqualität darzustellen, die einen gemeinsamen Bezugspunkt für die Auseinandersetzung mit Unterrichtsqualität für möglichst viele Fächer bietet - und durch die gemeinsame Sprache und Struktur die Möglichkeit schafft, Korrekturen und Ergänzungen über Fächer hinweg zu erarbeiten. Durch die integrative Betrachtung generischer und fachspezifischer Dimensionen wird dabei dem Umstand Rechnung getragen, dass Unterrichtsqualität stets ein Amalgam aus

\footnotetext{
2 Bei dem international gebräuchlichen Begriff Frameworks handelt es sich um Listen von als zentral erachteten Dimensionen von Unterrichtsqualität. Sobald konkrete Annahmen zu Zusammenhängen dieser Dimensionen untereinander oder zu Außenkriterien erfolgt, handelt es sich um ein Modell (siehe auch Praetorius et al. 2020b).
} 
Tab. 1 Dimensionen, Subdimensionen und fachspezifische Ergänzungen in der Version des erweiterten Syntheseframeworks von Praetorius et al. (2020a)

\section{Auswahl und Thematisierung von Inhalten und Fachmethoden}

I.1 Auswahl von bedeutungsvollen, dem Lernstand angemessenen Inhalten sowie Fachmethoden

I.2 Motivierung von Inhalten sowie Fachmethoden

I.3 Strukturierung der thematisierten Inhalte sowie Fachmethoden

I.4 Akkuratheit und Korrektheit der thematisierten Inhalte sowie Fachmethoden

Fachspezifische Ergänzungen Naturwissenschaften: Adäquate didaktische Reduktion unter Berücksichtigung zukünftiger fachlicher Lernschritte

\section{Kognitive Aktivierung}

II.1 Auswahl fachlich gehaltvoller und auf das kognitive Niveau der Schüler*innen abgestimmter Aufgaben

II.2 Einsatz fachlich gehaltvoller Aufgaben

II.3 Unterstützung der kognitiven Aktivität der Schüler*innen

II.4 Unterstützung des metakognitiven Lernens der Schüler*innen anhand kognitiv aktivierender Aufgaben

Fachspezifische Ergänzungen Naturwissenschaften: Kooperatives Arbeiten zur zielführenden Aktivierung der Schüler*innen

Fachspezifische Ergänzungen Sport: Berücksichtigung der Pädagogischen Perspektiven des Sports; Kognitive Verarbeitung von Bewegungshandlungen; Motorische Aktivierung

\section{Unterstützung des Übens}

III.1 Unterstützung bei der Festigung von Lern- und Anwendungsprozessen

III.2 Konstruktiver Umgang mit Fehlern und Schwierigkeiten von Schüler*innen beim Üben

Fachspezifische Ergänzungen: -

\section{Formatives Assessment}

IV.1 Klare Ausrichtung der Beurteilung auf die zu erlernenden Kompetenzen

IV.2 Regelmäßige Überprüfung des Verständnisses der Schüler*innen

IV.3 Qualitativ hochwertiges Feedback an die Schüler*innen

IV.4 Nutzung des Feedbacks als Grundlage für die Ausrichtung des weiteren Unterrichts

Fachspezifische Ergänzungen: -

\section{Unterstützung des Lernens aller Schüler*innen}

V.1 Bereitstellung eines Lernumfelds, das produktives Verhalten fördert

V.2 Differenzierung und Adaptivität

V.3 Förderung der aktiven Mitwirkung von allen Schüler*innen

Fachspezifische Ergänzungen Geschichte: Förderung des Unterrichtsdiskurses

\section{Sozio-emotionale Unterstützung}

VI.1 Beziehung zwischen Lehrperson und Schüler*innen

VI.2 Beziehung der Schüler*innen untereinander

Fachspezifische Ergänzungen Naturwissenschaften: Umgang mit Angst oder Ekel beim Lernen unter Gefährdungspotenzial oder an Lebewesen

\section{Klassenführung}

VII.1 Verhaltensmanagement

VII.2 Zeitmanagement

Fachspezifische Ergänzungen Naturwissenschaften \& Sport: Raum- und Materialmanagement; Gewährleistung der Sicherheit 
Fachinhalten, deren Didaktik und pädagogischen sowie psychologischen Aspekten darstellt (Reusser und Pauli 2021\#; Seidel et al. 2021\#).

Wir verfolgen mit der Entwicklung und Weiterentwicklung des Syntheseframeworks eine weitere Zielsetzung: Wie oben beschrieben, fokussiert die empirische Forschung zu Unterrichtsqualität auf eine Verknüpfung von Lehren und Lernen (siehe auch Decristan et al. 2020). Viele Frameworks oder Modelle von Unterrichtsqualität sind aber stark an Lernergebnissen der Schüler*innen in Form von Outcomes orientiert und nehmen die Lernprozesse - also die intraindividuelle Entwicklung von kognitiven Systemen - wenig in den Blick (Renkl 2012). Es wird entsprechend nicht oder nur wenig beachtet, wie sich Lernende mit welchen Aufgaben in welchen Repräsentationsformaten mental auseinandersetzen, wenn sie ihre Kompetenzen weiterentwickeln. Beispielsweise finden die Erkenntnisse aus der Expertiseforschung kaum Berücksichtigung, die nicht nur die Notwendigkeit einer intensiven Auseinandersetzung mit Lerngegenständen betont, sondern auch die NichtLinearität von Lernprozessen, z. B. die größeren Schwierigkeiten von ,intermediates“ (fortgeschrittenen Lernenden) bei der Wissensnutzung gegenüber Anfängern (z. B. Schmidt und Boshuizen 1993). Auch andere Forschungsrichtungen, die sich mit unterschiedlichen Akzenten - mit Lernprozessen befassen, werden in der Unterrichtsqualitätsforschung so gut wie nicht rezipiert, z. B. im Zusammenhang mit digitalem Lernen (z. B. dem ICAP-Model von Chi und Wylie 2014) oder der Bearbeitung von ausgearbeiteten Lösungsbeispielen (z.B. Renkl 2017). Es gibt Ausnahmen, in denen Lernprozesse und Unterrichtsqualität gezielt aufeinander bezogen werden. Hier ist insbesondere die Forschung zu den Unterrichtschoreographien zu nennen, die maßgeblich von Fritz Oser beeinflusst wurde und in verschiedenen Fachdidaktiken aufgenommen und weiterentwickelt wurde, insbesondere in den naturwissenschaftlichen Fächern (Oser und Baeriswyl 2001; Gräsel et al. 2021). Das Besondere dieses Ansatzes ist, dass er bestimmte kognitive Prozesse vorsieht, die in einer bestimmten Reihenfolge erfolgen müssen, um das jeweilige Lernziel zu erreichen. Wie diese kognitiven Prozesse (die „Tiefenstruktur") an der Oberfläche (der „Oberflächenstruktur“) realisiert werden, kann unterschiedlich sein - ähnlich wie eine schriftlich festgelegte Choreographie von Tänzer*innen unterschiedlich in einen Tanz übersetzt wird. Ein anderer Ansatz, der diese Verknüpfung in den Blick nimmt, ist die Differenzierung zwischen unterrichtlichem Angebot und dessen Nutzung durch die Schüler*innen (für einen Überblick zu Angebots-NutzungsModellen, siehe Vieluf et al. 2020). Die (Weiter-)Entwicklung des Syntheseframeworks soll auch dazu dienen, diese Verknüpfung von unterrichtlichen Angeboten und Lernprozessen wieder stärker in den Fokus der Unterrichtsforschung zu rücken (Charalambous und Praetorius 2020), wenngleich diese Entwicklung noch in den Anfängen steckt.

Im vorliegenden Beitrag wird nun der Versuch unternommen, zentrale Aspekte der einzelnen Beiträge des Themenheftes zusammenfassend darzustellen sowie erste Ideen für eine zukünftige Weiterentwicklung der Frage nach der Fachspezifität im Allgemeinen sowie des Syntheseframeworks im Speziellen vorzuschlagen. Dabei legen wir bewusst Schwerpunkte auf aus unserer Sicht besonders auffällige Fachunterschiede und Gemeinsamkeiten. 


\section{3 Übersicht über die in den Beiträgen der Fächer thematisierten Dimensionen}

Im Folgenden fassen wir zentrale Aussagen der neun fachdidaktischen Einzelbeiträge des aktuellen Themenheftes zu den sieben Dimensionen des Synthesebeitrags und seiner Ergänzungen knapp zusammen (für eine Übersicht über die Dimensionen sowie die im vorangegangenen Thementeil erarbeiteten Ergänzungen siehe Tab. 1). Es ist uns wichtig darauf hinzuweisen, dass diese Zusammenfassung keinen Anspruch auf Vollständigkeit erhebt, sondern sich ausschließlich an den konkreten Texten orientiert, die natürlich unterschiedliche Akzente gesetzt haben und auf unterschiedlichen fachspezifischen Forschungsständen aufbauen konnten.

\subsection{Auswahl und Thematisierung von Inhalten und Fachmethoden}

Gerade den Fachdidaktiken war und ist es ein großes Anliegen, Unterrichtsqualität unter der Perspektive zu betrachten, ob die Auswahl und Thematisierung fachlicher Inhalte angemessen und begründet erfolgt und ob adäquate Fachmethoden verwendet werden (z.B. die Art und Weise der Einbindung naturwissenschaftlicher Denk- und Arbeitsweisen). Zusammenfassend über die Beiträge der verschiedenen Fächer wird deutlich, dass die fachliche Richtigkeit und Stimmigkeit in vielen Beiträgen als wichtiger Aspekt von Unterrichtsqualität hervorgehoben wird (Deutsch, Englisch, Geschichte, Sport, Biologie, Mathematik). Auch die Wahl geeigneter und fachlich begründeter Methoden/Vorgehensweisen wird durchgängig als wichtig thematisiert. In den musisch-ästhetischen Fächern wird die Auswahl von Artefakten und Lernmaterial als bedeutsam neben den Inhalten und Methoden angesprochen (Musik, Kunst). Im Sport wird darauf verwiesen, dass einige Inhalte des Sportunterrichts wenige Überschneidungen mit anderen Fächern haben und dies in besonderer Weise inhaltliche und methodische Spezifität mit sich bringt (z. B. für den Bereich des motorischen Lernens). Das Fach Religion verweist auf die inhaltliche Besonderheit der Transzendenz sowie auf die Bedeutsamkeit existenzieller Bezüge, die Unterricht vor besondere Herausforderungen stellt. Etwas anders gelagert stellt sich das Vorgehen des Beitrags aus der Geschichtsdidaktik dar: Hier wird der Versuch unternommen, Unterrichtsqualitätsdimensionen explizit mit einem Kompetenzmodell in Zusammenhang zu bringen und damit fachlich zu begründen.

\subsection{Kognitive Aktivierung}

Kognitive Aktivierung wird in allen Fächer-Beiträgen im vorliegenden Themenheft als bedeutsam herausgestellt. In einzelnen Beiträgen (Biologie, Geschichte) wird zudem auf den hohen Grad der Fachspezifität kognitiver Aktivierung im Vergleich zu anderen Dimensionen der allgemeinen Unterrichtsqualitätsforschung hingewiesen. Für das Fach Deutsch wird eine Dominanz kognitiver Aktivierung in empirischen Untersuchungen im Vergleich zu anderen Dimensionen thematisiert; in diesen Studien wird kognitive Aktivierung fachbezogen oder fachspezifisch erfasst. Darüber hinaus werden in einigen Beiträgen notwendige Ergänzungen und Anpassungen diskutiert (Kunst, Sport, Musik, Englisch): Für das Fach Kunst erscheint eine Er- 
weiterung $\mathrm{zu}$ „kognitiver und emotionaler Aktivierung“ notwendig, um der engen Verschränkung von rationalen und ästhetischen Erfahrungen gerecht zu werden. Die Bedeutung von kognitiv-emotionaler Aktivierung wird in Bezug auf Unterricht, der auf eine subjektive Involviertheit der Schüler*innen abzielt, auch in der literaturdidaktischen Diskussion als relevant herausgestellt (Hesse 2021). Für das Fach Sport wurde im Vorgängerheft aufgrund der engen Verknüpfung von Kognition und Motorik eine Erweiterung hin zu kognitiv-motorischer Aktivierung vorgeschlagen (Hermann und Gerlach 2020). Im vorliegenden Heft wird dieser Vorschlag nicht aufgegriffen (Richartz und Kohake 2021\#) und vielmehr auf die Relevanz kognitiver Aktivierung für lediglich ausgewählte Inhalte im Sport hingewiesen und zeitgleich eine ergänzende, separate Dimension gefordert, die motorische und spieltaktische Unterrichtsaspekte explizit thematisiert. Eine analoge Argumentation wie bei Herrmann und Gerlach (2020) erfolgt im Musik-Beitrag. In diesem Beitrag wird zudem wie in Kunst auch auf die hohe Relevanz ästhetischer Aktivierung verwiesen, gleichzeitig aber hinterfragt, inwiefern diese als eine ergänzende Facette der kognitiven Aktivierung gesehen werden sollte oder eine zusätzliche fachspezifische Dimension darstellt. Im Englisch-Beitrag schließlich wird die Erweiterung hin zu einer kommunikativ-kognitiven Aktivierung diskutiert mit dem Verweis auf die zentrale, identitätsstiftende Rolle von Kommunikation im Fremdsprachenunterricht.

\subsection{Unterstützung des Übens}

Die Unterstützung des Übens wird in einigen Beiträgen nicht erwähnt bzw. nur sehr kurz ausgeführt (Deutsch, Religion, Biologie, Mathematik). Im Englisch-Beitrag wird spezifisch für den Spracherwerb die Rolle des lernwirksamen und motivierenden Umgangs mit Fehlern beim Üben thematisiert. Im Fach Kunst gibt es Ansätze, die das Üben nur für wenige Unterrichtsinhalte als Dimension von Unterrichtsqualität sehen und es für andere kritisch betrachten (vgl. Kirchner und Kirschenmann 2015). Im Fach Sport wird das Üben und die Unterstützung des Übens für die bewegungsorientierten Lernziele als konstitutiv betrachtet, insbesondere für die Prozeduralisierung des spieltaktischen Lernens, nicht hingegen bei Zielen, die auf reflexive Handlungsfähigkeit abzielen. In der Musik kommt dem Üben in den musikalischen Praxisphasen ebenfalls ein hoher Stellenwert zu. Auch im Fach Geschichte wird die Fachspezifik des Übens betont, die u. a. darin besteht, dass durch wiederholendes Üben gelernt wird, historische Kategorien und Konzepte zu erkennen, aber auch ihre epochen- und kulturspezifischen Veränderungen wahrzunehmen.

\subsection{Formatives Assessment}

Von den neun einbezogenen Fachdidaktiken im vorliegenden Themenheft wurde formatives Assessment in drei Beiträgen nicht erwähnt oder nur indirekt adressiert (Englisch, Religion, Biologie). In den Beiträgen, in denen formatives Assessment thematisiert wird, werden die aufgegriffenen Aspekte vielfach anderen Dimensionen zugeordnet (z.B. konstruktiver Unterstützung). Die erwähnten Aspekte stellen typische Teilbereiche formativen Assessments dar: So wird für das Fach Kunst angegeben, dass es eine explizite Auseinandersetzung zu Feedback und transparenten 
Bewertungen gibt. Auch im Sport wird explizit auf die bedeutsame Rolle lernbegleitenden Feedbacks verwiesen, insbesondere für das Bewegungslernen. Im MusikBeitrag wird als fachspezifisches Element das dort vorherrschende Spannungsfeld zwischen „Anleitung und Eigen-Sinn“ hervorgehoben. Zwei weitere Beiträge verweisen ohne weitere Ausführungen lediglich auf den Begriff formatives Assessment oder den Teilbereich Feedback (Geschichte, Mathematik).

\subsection{Unterstützung des Lernens aller Schüler*innen}

In der Unterrichtsforschung wird der Umgang mit Heterogenität und die Anpassung des Unterrichts an die Lernvoraussetzungen von Schüler*innen seit vielen Jahren einhellig als Voraussetzung für eine wirkungsvolle Unterstützung der Schüler*innen diskutiert (vgl. Gräsel et al. 2017; Klieme und Warwas 2011), auch wenn einige Frameworks für Unterrichtsqualität diese Dimension nicht eigenständig ausweisen (siehe aber z.B. CLASS, Pianta et al. 2012; TRU, Schoenfeld 2018). In manchen fachdidaktischen Beiträgen findet sich ein Übereinstimmen mit dieser Dimension im Sinne der Differenzierung und der individuellen Förderung, ohne weiterführende Erläuterungen (Sport, Religion, Biologie). In einigen Beiträgen wird der Vorschlag des geschichtsdidaktischen Beitrags von Zülsdorf-Kersting (2020) im Vorgängerheft aufgegriffen, die Diskursqualität im Unterricht als Subdimension zu ergänzen (Englisch, Musik, Mathematik; zu deren Bedeutung, siehe auch Reusser und Pauli 2021\#). In der Biologie wird zudem insbesondere die Individualisierung des Lernens hervorgehoben (z. B. durch Wahlmöglichkeiten von Medien); in der Geschichte werden die sprachlichen Voraussetzungen der Lernenden als bedeutsam thematisiert. Für den Kunstunterricht wird die Orientierung an individuellen Interessen betont. In der Kunst wird zusätzlich auf die Beteiligung und Partizipation aller Schüler*innen als Qualitätsmerkmal verwiesen, das Raum gibt für individuelle kreative Prozesse.

\subsection{Sozio-emotionale Unterstützung}

Die sozio-emotionale Unterstützung wird in einigen hier berücksichtigten Fachdidaktiken entweder explizit (Sport) oder implizit (Biologie, Mathematik) thematisiert. Die Ausführungen weisen darauf hin, dass sozio-emotionale Unterstützung und die Unterstützung kognitiver Lernprozesse oftmals zusammengenommen wird (Religion), während im Beitrag aus dem Fach Deutsch explizit eine Differenzierung der beiden erfolgt. Für guten Geschichtsunterricht wird postuliert, dass er eine Synthese zwischen kognitiver Aktivierung und sozio-emotionaler Unterstützung erfordert - insbesondere wegen der (starken) Irritationen der Schüler*innen, die bei einer Beschäftigung mit historischen Sachverhalten auftreten können. In der Kunst wird sozio-emotionale Unterstützung in die allgemeinere Diskussion eines „kreativitätsfördernden Klimas“ einbezogen. Im Sport und in der Musik wird angesprochen, dass die Vermeidung von Beschämung der Schüler*innen eine wichtige Aufgabe der sozio-emotionalen Unterstützung sei. Der Beitrag aus dem Sport betont, dass dies in anderen Fächern ebenso gilt, dort aber wenig Berücksichtigung findet: Selbst-Exposition, starke soziale Bezugsnormen und Etikettierungsprozesse treten in einigen Fächern vielleicht verstärkt auf, sind aber eben nicht ausschließlich 
dort für das Erleben (fehlender) sozio-emotionaler Unterstützung relevant. Auffallend ist schließlich, dass die fachdidaktischen Arbeiten auf die Beziehung zwischen Lehrperson und Schüler*innen fokussieren und - abgesehen von der Thematik der Scham - die Beziehungen unter den Peers, die ja für die sozial-emotionale Situation von Schüler*innen ebenfalls relevant sind, nicht nennen.

\subsection{Klassenführung}

Klassenführung wird in einigen Fächern nicht explizit erwähnt (Mathematik) oder ohne weitere Ausführungen als bedeutsam genannt (Deutsch, Englisch, Religion, Sport). Zudem zeigt sich, dass trotz der hohen Popularität von Klassenführung kein einheitliches Verständnis derselben vorherrscht. So wird im Biologiebeitrag neben dem im Syntheseframework thematisierten Verhaltens- und Zeitmanagement auch Klarheit der Klassenführung zugeordnet. Im Geschichtsbeitrag wird vornehmlich der Fokus auf Zielklarheit und Struktur gelegt. In Kunst und Musik hingegen wird hinsichtlich der Klassenführung auf die Spezifika des jeweiligen Fachs verwiesen. So wird im Kunstbeitrag hervorgehoben, dass aufgrund der Relevanz ästhetischer Prozesse Offenheit und Komplexität notwendig sind, was wiederum erfordert, dass Anweisungen zum Teil unspezifisch erfolgen. Für die Musik wird die im Syntheseframework von Praetorius et al. (2020a) hervorgehobene notwendige Ergänzung von Klassenführung um eine Subdimension zu Raum- und Materialmanagement dezidiert unterstützt. Zudem wird darauf verwiesen, dass weitere bislang nicht beachtete Aspekte eine Rolle für die Klassenführung im Fach Musik spielen (u.a. aufgrund der spezifischen Anforderungen, die durch gemeinsames Musizieren entstehen) und entsprechend deutliche fachspezifische Anteile der Klassenführung zu konstatieren sind. Bezugnehmend auf die Überlegungen von Praetorius et al. (2020a) wird daher vorgeschlagen, anstelle von Klassenführung von „Klassenführung in Musik“ zu sprechen, um die Verschiebung in der Bedeutung der Konstrukte zu verdeutlichen.

\subsection{Zusammenfassung der Befunde}

Zusammengenommen lässt sich mit Blick auf die Dimensionen des erweiterten Syntheseframeworks zunächst einmal feststellen, dass in Bezug auf die vier Dimensionen, die direkt am Lernprozess der Schüler*innen orientiert sind, zwei Dimensionen in allen Fächern als bedeutsam thematisiert und dabei zum Teil fachspezifisch ausdifferenziert werden (Auswahl und Thematisierung von Fachinhalten und Fachmethoden; kognitive Aktivierung). Zwei Dimensionen hingegen werden nur in ausgewählten Fächer-Beiträgen thematisiert (Unterstützung des Übens; formatives Assessment) und dabei entweder auf deren Bedeutsamkeit für lediglich einige Inhalte verwiesen (Unterstützung des Übens) und/oder insgesamt wenig konkret beschrieben (Unterstützung des Übens; formatives Assessment). Die drei Dimensionen, die indirekt mit allen Schritten des Lernprozesses verknüpft sein sollten, werden entweder in allen Fächern als bedeutsam identifiziert, aber nicht weiter ausgeführt (Unterstützung des Lernens aller Schüler*innen) oder lediglich in einigen Fächern erwähnt und dabei konzeptuell heterogen verstanden bzw. fachspezifisch ausgeführt (Klassenführung) oder es werden Teilaspekte nicht explizit berücksich- 
tigt (Beziehung von Schüler*innen untereinander in der Dimension sozio-emotionale Unterstützung). Betrachtet man die Adaptionen, die in den Beiträgen vorgeschlagen werden, dann lassen sich Unterschiede zwischen den Fächern erkennen: Keine oder wenige Adaptionen wurden insbesondere von den Fächern vorgeschlagen, die in der allgemeinen Unterrichtsforschung traditionell eine große Rolle spielen (Mathematik; Naturwissenschaften). In diesen Beiträgen wurde entsprechend auch der Schwerpunkt nicht oder nicht vorrangig auf eine Überprüfung der Übereinstimmung mit dem Syntheseframework gesetzt. Für die anderen Fächer finden sich erwartungsgemäß mehr Vorschläge für fachliche Anpassungen. Zukünftig wäre nun besonders spannend, diese Adaptionsvorschläge dahingehend zu überprüfen, ob sie lediglich für ein einzelnes Fach, für bestimmte Fächergruppen oder aber gegebenenfalls für alle Fächer von Bedeutung sind. Schließlich gilt zu beachten, dass aufgrund der jeweils individuellen Auseinandersetzung mit dem erweiterten Syntheseframework in den einzelnen Beiträgen keine gemeinsam geteilten und ausgehandelten Begriffe bzw. Dimensionen erarbeitet wurden. Entsprechend wird in verschiedenen Beiträgen die Diskussion um notwendige Anpassungen etwas unterschiedlich geführt. Für die weitere Konsensbildung darüber, inwieweit Anpassungen für ein einzelnes Fach, für bestimmte Fächergruppen oder alle Fächer Gültigkeit haben, wären vertiefte gemeinsame Diskussionen - beispielsweise im Rahmen von Fachtagungen - wünschenswert. Wir würden uns freuen, wenn wir mit unserem Themenheft und diesem einleitenden Beitrag dazu anregen könnten.

\section{Vertiefende Diskussion ausgewählter Dimensionen}

Im Folgenden möchten wir zwei Dimensionen, kognitive Aktivierung und Unterstützung des Übens, noch etwas differenzierter betrachten. Wir haben diese Dimensionen zum einen ausgesucht, weil andere Systematiken der Unterrichtsqualität zwischen ihnen nicht unterscheiden und wir damit die Bedeutung unterstreichen wollen, sie zu differenzieren. Zum anderen werden sie in den Beiträgen aus den Fachdidaktiken unterschiedlich betrachtet: Kognitive Aktivierung hat für alle Fächer eine große Bedeutung, aber es werden auch etliche Anpassungen und Ergänzungen formuliert. Die Unterstützung des Übens wird in den Einzelbeiträgen eher randständig bzw. allgemein behandelt, hier sehen wir einen großen Bedarf für eine theoretische und empirische Weiterentwicklung.

\subsection{Kognitive Aktivierung als theoretisch unterspezifizierte Dimension}

Kognitive Aktivierung hat sich nach ihrer Einführung in der deutschen TIMSS-Videostudie (siehe Klieme et al. 2001) in der deutschsprachigen Unterrichtsforschung rasch als zentral bearbeitete Dimension von Unterrichtsqualität etabliert. Kognitive Aktivierung kann nach Klieme et al. (2009) wie folgt definiert werden: „As a seminal feature of the deep structure ... of classroom learning, it relates to any observable pedagogical practice and pattern on the surface level of instruction that encourages students to engage in (co-)constructive and reflective higher-level thinking and thus to develop an elaborated, content-related knowledge base“ (S. 140-141). Während 
auf dieser abstrakten Ebene überwiegend Einigkeit herrscht, was kognitive Aktivierung meint, zeigt ein Blick auf ihre konkreten Operationalisierungen eine große Spannbreite (Hugener 2008; Praetorius et al. 2018). Dies liegt sicherlich insbesondere daran, dass der Begriff kognitive Aktivierung in der Unterrichtsforschung als Sammelbegriff für mehrere mittels exploratorischer Faktorenanalyse als zusammengehörig identifizierte Skalen eingeführt wurde (siehe Klieme et al. 2001), die sich ihrerseits unter anderem an konstruktivistischen Vorstellungen über Lernprozesse orientierten (Klieme 2019; für die ursprünglichen Skalen aus Sicht der Schüler*innen, siehe Gruehn 2000). Seitdem fand nur wenig Theorieentwicklung statt, wenn man von der Spezifikation der Annahme absieht, dass kognitive Aktivierung (als unterrichtliches Angebot) zu einer tieferen Informationsverarbeitung (als Nutzung des Angebots durch die Schüler*innen) und so zu höheren Schüler*innenleistungen führen sollte (Klieme und Rakoczy 2008). Eine weiterführende theoretische Basis wäre jedoch eine Voraussetzung, um Anpassungs- und Ergänzungsvorschläge in einzelnen Fächern zu diskutieren (siehe Martin-Raugh et al. 2016; siehe auch Praetorius et al. 2020a). Ergebnisse könnten eine Erweiterung kognitiver Aktivierung über Fächer hinweg oder aber lediglich für einzelne Fächer hin zu einer kognitivmotorischen, kognitiv-ästhetischen oder kommunikativ-kognitiven Aktivierung sein oder aber auch die Entscheidung, diese ergänzenden Aspekte nicht als Teil kognitiver Aktivierung, sondern als eigenständige Dimensionen zu behandeln sein (siehe auch Richartz und Kohake 2021\#; Kranefeld 2021\#). Für die Weiterentwicklung der theoretischen Basis ist unserer Einschätzung nach ein intensiver interdisziplinärer Austausch von generischen sowie fachspezifischen Unterrichtsforschenden erforderlich. In diesem könnte erstens in Betracht gezogen werden, kognitive Aktivierung stärker vom Lernprozess der Schüler*innen her zu denken (siehe Abschnitt 5.2) und dabei zweitens die Anregung von Seidel et al. (2021\#) aufgegriffen werden, ein Set standardmäßig vorkommender Situationen für kognitive Aktivierung zu entwickeln mit spezifischen Anforderungs- und Zielkonstellationen für zentrale fachliche Inhalte. Ein solches Set würde es ermöglichen, sich verstärkt mit möglichen Wegen zur Umsetzung kognitiver Aktivierung im Unterricht auf eine konkrete Art und Weise auseinanderzusetzen und diese zwischen Fächern zu vergleichen. Sinnvoll erscheint es dabei auch, verschiedene Umsetzungsmöglichkeiten miteinander zu vergleichen, die sich bzgl. der Oberflächen-, nicht aber der Tiefenstruktur von Unterricht (Vieluf et al. 2020) unterscheiden. Darüber hinaus würde es sich anbieten, neben gelungenen Umsetzungsmöglichkeiten auch suboptimale Optionen zu skizzieren (Scheerens 2015), da diese ebenfalls sowohl für die Forschung als auch für die praktische Umsetzung hoch informativ sind. Ähnlich wie Seidel et al. (2021\#) stellen Trautwein et al. (2021\#) in Frage, inwiefern eine Separierung kognitiver Aktivierung von geeigneten Inhalten sinnvoll ist (die im oben dargestellten Syntheseframework eine separate Dimension darstellt) (siehe auch Lindmeier und Heinze 2020).

Diskussionswürdig erscheint uns für eine zukünftige Auseinandersetzung mit kognitiver Aktivierung unter anderem der notwendige Auflösungsgrad, also die Frage ob die theoretische Arbeit auf Ebene der Fächer oder aber auf Ebene konkreter Inhalte erfolgen sollte. Kirschner et al. (2017) sowie Seidel et al. (2021\#) argumentieren für letzteres, während Dreher und Leuders (2021\#) argumentieren, dass die Spezifikation generischer Aspekte um fach- und inhaltsspezifische Aspekte durch 
die den Unterricht einschätzenden Beobachtenden erfolgen sollte, anstelle die entsprechenden Instrumente zu überladen. Und schließlich erscheint es lohnenswert zu durchdenken, für welche Ziele, Inhalte und Fachmethoden kognitive Aktivierung eine angemessene Qualitätsdimension des Unterrichts darstellt. Wie von Richartz und Kohake (2021\#) für das Fach Sport ausgeführt, ist dies beispielsweise bei der Förderung des Bewegungskönnens nicht der Fall bzw. sogar kontraproduktiv.

\subsection{Die unterschätzte Bedeutsamkeit der Unterstützung des Übens}

Im (erweiterten) Syntheseframework wird - im Unterschied zu vielen anderen Systematiken (z. B. den drei Basisdimensionen) - die Unterstützung des Übens als eigene Qualitätsdimension herausgestellt, die auf die Konsolidierung von Gelerntem abzielt, beispielsweise durch Unterstützung bei Schwierigkeiten (Praetorius und Charalambous 2018). Durch den Fokus auf die drei Basisdimensionen im Anschluss an die Publikation der deutschen TIMSS-Videostudie (Klieme et al. 2001) geriet die Bedeutung des Übens gegenüber der kognitiven Aktivierung in der Forschung in den Hintergrund. Das Syntheseframework griff diese Dimension aus mehreren Gründen wieder als eigenständig auf: Die Unterstützung des Übens umfasst die lernförderliche Platzierung des Übens im Unterricht, die Auswahl und Sequenzierung geeigneter Übungsaufgaben und das Geben von Feedback, wobei im Syntheseframework (siehe Tab. 1) dem konstruktiven Umgang mit Fehlern und Schwierigkeiten ein besonderer Stellenwert zukommt. Üben ist für das Lernen von zentraler Bedeutung; daher sollte die Qualität des Übens sowie Möglichkeiten zu deren Unterstützung explizit in den Forschungsfokus genommen werden. In der Forschung zu Unterrichtsqualität ist das - im Gegensatz zur Instruktionsforschung (siehe unten) gegenüber der kognitiven Aktivierung etwas in den Hintergrund geraten. Wenn Unterricht mit Kategorien nicht nur beschrieben werden soll, sondern sich durch die Beschreibung auch Anhaltspunkte für seine Verbesserung ergeben sollen, dann ist es von Bedeutung die Unterstützung des Übens als eigene Dimension zu analysieren. Die Analyse von Übungsaufgaben, ihrer Bearbeitung und angemessenen Rückmeldungen bietet auch die Möglichkeit, die Unterrichtsqualitätsforschung wieder stärker mit Erkenntnissen der Lern- und Instruktionsforschung zu verbinden (siehe auch Abschnitt 5.2). Zusammengenommen geben die Ausführungen aus den Fachdidaktiken Hinweise für eine Weiterentwicklung der Dimension unter zwei Perspektiven: Erstens ist für deren Weiterentwicklung vielleicht weniger die Frage von Bedeutung, ob es sich um eine generische oder eine fachspezifische Dimension handelt - hier zeichnet sich ein ,sowohl generisch als auch inhaltsabhängig akzentuiert“ ab. Bedeutsamer wäre es für die Unterrichtsforschung, sich stärker mit der Frage zu befassen, wie eine Unterstützung guten Übens theoretisch gefasst und für verschiedene Inhaltsbereiche ausdifferenziert und realisiert werden kann. Für diese Arbeiten kann man sich an älteren und jüngeren Ansätzen der Instruktionsforschung orientieren, die sich mit Expertise und Expertiseentwicklung befassen. Dazu gehören die klassischen Arbeiten der deliberate practice, die die Gestaltung von Lerngelegenheiten und Rückmeldungen über eine längere Phase thematisieren (Ericsson 1996; Ericsson und Harwell 2019). Deliberate practice beinhaltet das auf Verbesserung der eigenen Fähigkeiten abzielende Üben auf einem Niveau, das Anstrengung erfordert. 
Die These von Ericsson (1996), wonach es in erster Linie das Ausmaß und die Qualität der deliberate practice ist, die Expert*innen von Nicht-Expert*innen unterscheidet, wird anhand neuerer empirischer Studien relativiert (z.B. Macnamara et al. 2014). Unbestritten ist aber, dass der Ansatz für den Erwerb vernetzten und gut organisierten Wissens sowie für den Fertigkeitserwerb von großer Bedeutung ist. Für deliberate practice ist das Setzen herausfordernder, aber erreichbarer Ziele (jenseits der Komfortzone), eine hohe Persistenz und Konsistenz beim Üben und vor allem Feedback durch Expert*innen konstitutiv. Dieses Konzept spielt in neueren Ansätzen der Unterrichtsforschung eine bedeutende Rolle, insbesondere im Ansatz der sustained learning opportunities (Hiebert und Stiegler im Druck). Dieser Ansatz postuliert einige Prinzipien für verstehensorientiertes Üben, z. B. eine Phase des productive struggling zu Beginn des Lernens oder die Bedeutung des Herstellens und Explizierens von Wissensrelationen in der Lerngelegenheit, z. B. durch Analogien, Vergleiche, Erklärungen oder visuelle Repräsentationen. Eine Arbeit von Fries et al. (2020) expliziert die Bedeutung des Übens für den Erwerb und die Nutzung von begrifflichem Wissen in einer Domäne: Erst durch Konsolidierung und damit durch die wiederholte Nutzung von Wissen bei der Bearbeitung von Aufgaben und Problemen werden Relationen hergestellt zwischen (a) den Kernbegriffen (core concepts) einer Disziplin, (b) Konzepten und ihrer Darstellung in wichtigen Repräsentationsformen sowie (c) zwischen den Konzepten und der Wissensanwendung in konkreten Handlungssituationen. Durch die Realisierung einer geeigneten Unterstützung des Übens wird also der Fertigkeitserwerb ebenso unterstützt wie die Entwicklung eines gut strukturierten, eng vernetzten Wissens in einer Domäne. Aus der Transferforschung (Schwartz et al. 2005) ist darüber hinaus bekannt, dass gut strukturiertes Wissen und eng vernetztes Wissen die Voraussetzung für den Wissenstransfer sind. Zweitens wird das Üben (nicht nur) im Syntheseframework als bedeutsam für die Konsolidierung von Wissen betrachtet, das sich auf Fertigkeiten (skills) bezieht - und dabei ausdrücklich auch auf komplexe Fertigkeiten, welche die Anwendung deklarativen und prozeduralen Wissens aus verschiedenen Inhaltsbereichen auf konkrete Situationen erfordern. Der Beitrag aus der Sportdidaktik erweitert diese Funktionalität: Für den Bereich des Bewegungslernen ist das Üben nicht nur für die Konsolidierung, sondern von Beginn des Lernens an für das Bewegungslernen wichtig; die Eigenlogik des Bewegungslernens erfordert die Aufmerksamkeitslenkung auf das Ergebnis (z.B. Ball im Korb) und nicht auf die Steuerung des Ablaufs der Bewegung - dies $\mathrm{zu}$ berücksichtigen ist ein spezifischer unterrichtlicher Qualitätsaspekt. Eine ähnliche Kombination des Erwerbs und des Übens von Beginn des Lernprozesses an könnte auch für bestimmte musikalische Lernziele gelten, die ebenfalls im Bereich des sensomotorischen Lernens liegen. Dementsprechend könnte zumindest für sensomotorisches Lernen eine weitere Subdimension eingeführt werden, nämlich ein „erwerbsunterstützendes Üben“, das insbesondere für motorische Bereiche künftig weiter ausdifferenziert werden sollte. 


\section{5 Überlegungen zu einer Weiterentwicklung des Forschungsbereichs}

Abschließend folgen einige Überlegungen zu möglichen nächsten Schritten bei einer Auseinandersetzung mit der Fachspezifität von Unterrichtsqualität.

\subsection{Die komplexe Auseinandersetzung mit den Zielen von Unterricht}

Ziele werden in der bisherigen empirischen Unterrichtsforschung vorrangig als mehr oder weniger austauschbare Outcome-Variablen von Unterrichtsqualität betrachtet. Dies greift jedoch zu kurz. So müssten in der Forschung zunächst einmal neben den bislang sehr dominanten Wissenserwerbszielen weitere bedeutsame Ziele in den Blick genommen werden (z. B. Interesse am Fach; Lernen lernen; vertiefte Gesellschaftsreife ${ }^{3}$ ), auch wenn diese Ziele zum Teil schwer operationalisierbar sind (siehe Rothgangel 2021\#) und verschiedene Ziele ggf. auch konfligierend sind. Zudem müsste stärker expliziert werden, dass selbst innerhalb von Fächern in Bezug auf die besonders zentralen Ziele keine Einigkeit herrscht (Praetorius et al. 2020a). Die Erreichung dieser Ziele müsste zudem auch in längerfristig angelegten Studien überprüft werden anstelle der bislang üblichen querschnittlichen sowie längsschnittlichen Untersuchungen von Kurzzeiteffekten (in der Regel max. ein Schuljahr). Dabei müsste zudem berücksichtigt werden, dass einige Dimensionen von Unterrichtsqualität nur in Bezug auf bestimmte Ziele sinnvoll zu untersuchen sind (siehe Kranefeld 2021\#; vgl. Abschnitt 4.1). Entsprechend kann Unterrichtsqualität ohne eine Kenntnis der zu erreichenden Ziele kaum sinnvoll beurteilt werden. Auch über die konkrete Verknüpfung von Zielen und unterrichtlichen Prozessen zu deren Erreichung ist bislang nur sehr wenig bekannt (siehe auch Seidel et al. 2021\#). Dies ist umso bedeutsamer, da sich die Bildungsziele zwischen den Fächern zum Teil sehr deutlich unterscheiden und auch aus unterschiedlichen Bezugsdisziplinen heraus entwickelt wurden (z. B. in der Kunst aus dem klassischen Bildungsverständnis; in Deutsch und Religion aus unterschiedlichen Disziplinen zusammengestellt).

\subsection{Die Verknüpfung von Unterrichtsdimensionen und Lernprozessen}

Nimmt man den Anspruch der Lehr-Lernforschung im Allgemeinen sowie der Unterrichtsqualitätsforschung im Speziellen ernst, dann sollte eine enge theoretische sowie empirische Abstimmung zwischen Lehren und Lernen erfolgen (siehe auch Reusser und Pauli 2021\#). Dies wurde bereits in den 1970er und 1980er-Jahren gefordert (z. B. Brophy 1986; Doyle 1977; Gage und Needels 1989; Winne 1987), fand dann aber keinen breiten Eingang in die theoretische Auseinandersetzung mit und die empirische Forschung zu Unterrichtsqualität - zumindest über den Verweis auf Angebots-Nutzungs-Modelle als Rahmenmodell hinaus (siehe auch Abschnitt 2; für Ausnahmen siehe Hiebert und Stigler in Druck; Klauer und Leutner 2012; Oser und Baeriswyl 2001; Seidel und Shavelson 2007). Basierend auf dem erweiterten Syntheseframework von Praetorius et al. (2020a) haben Charalambous und Praetorius

\footnotetext{
3 Mit dem Begriff ist breite Allgemeinbildung gemeint, welche die „Grundlage zur Lösung anspruchsvoller Aufgaben in der Gesellschaft“" darstellt (Eberle et al. 2008, S. 18).
} 


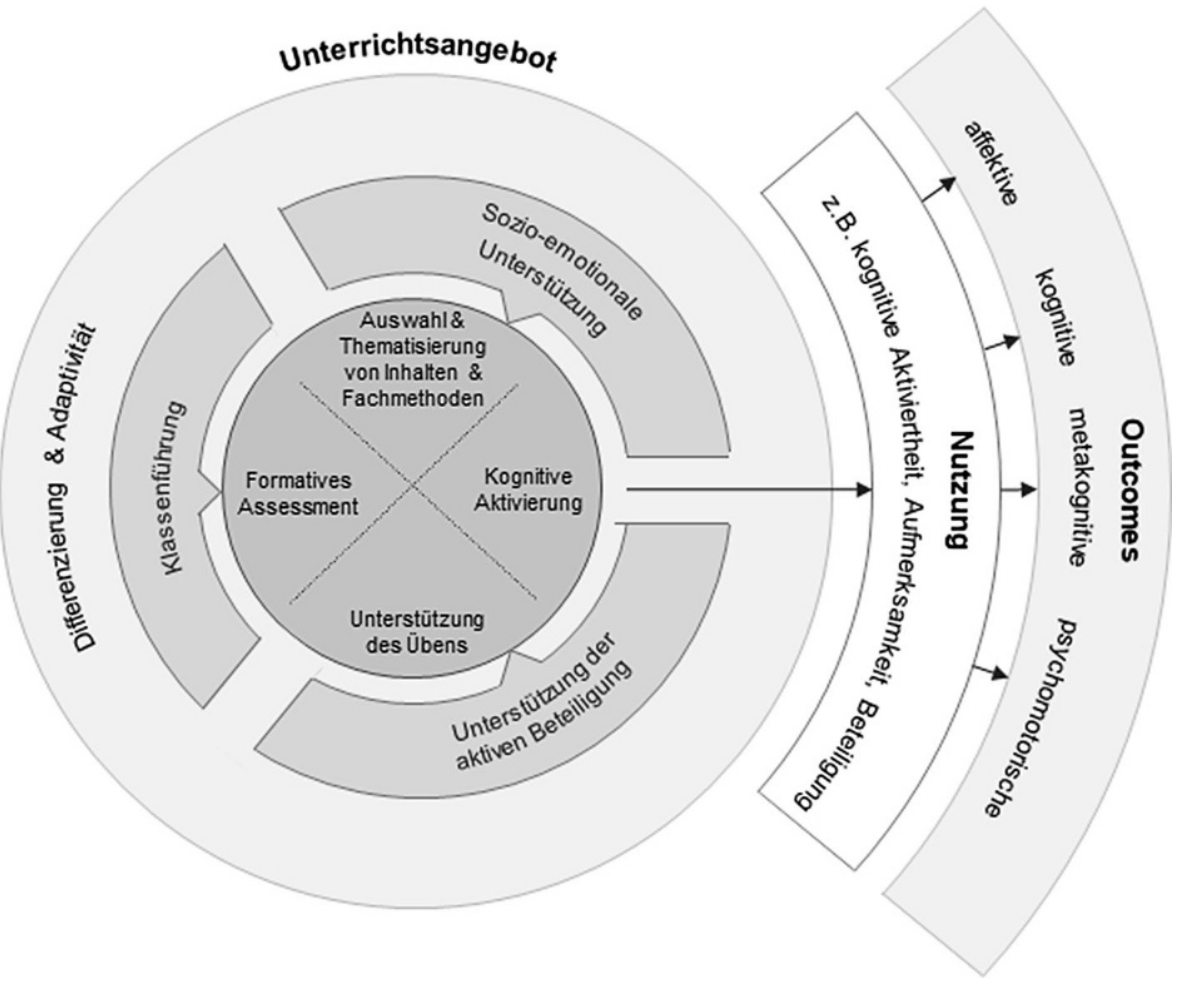

Abb. 1 Das auf Basis des erweiterten Syntheseframeworks entwickelte MAIN-TEACH-Modell (nach Charalambous und Praetorius 2020)

(2020) ein Modell entwickelt, das eine solche stärkere Verknüpfung zwischen Unterrichtsdimensionen, Lernprozessen und Outcomes ermöglichen soll: das MAINTEACH-Modell (multi-layered and integrated in conceptualizing the quality of teaching; siehe Abb. 1).

Das Modell nimmt Bezug auf existierende Ansätze zur Erforschung von Unterrichtsqualität und entwickelt diese weitere. Dadurch können zum einen bestehende Ansätze in das Modell integriert werden (z. B. die zentralen Aspekte des Modells der drei Basisdimensionen, aber auch diejenigen des Dynamic Models for Educational Effectiveness; Creemers und Kyriakides 2008) und zum anderen wird es so ermöglicht, diese unterschiedlichen Ansätze aufeinander zu beziehen und so eine mögliche Basis für die Zusammenarbeit über Arbeitsgruppen hinweg zu bieten (siehe das sich aktuell in Druck befindliche Special Issue in Studies in Educational Evaluation zu „Merits and limitations of researching teaching quality more synergistically“").

Das Modell integriert explizit generische und fachspezifische Aspekte von Unterrichtsqualität. So enthält beispielsweise die Dimension kognitive Aktivierung diejenigen Aspekte, die sowohl in generischen Ansätzen als auch in fachspezifischen Ansätzen als bedeutsam erachtet werden (für weitere Informationen siehe Praetorius und Charalambous 2018; Praetorius et al. 2020a). 
Das Modell erlaubt zudem eine Relationierung der Unterrichtsdimensionen untereinander, was einen entscheidenden Vorteil gegenüber der reinen Auflistung von Dimensionen darstellt, da es der Beliebigkeit der Ausdifferenzierung von mehr oder weniger Dimensionen (siehe Helmke 2017) entgegenwirkt. Erste Vorschläge für solche Relationierungen wurden bereits in den 1970er-Jahren vorgenommen (siehe Openshaw und Clarke 1970), seitdem aber kaum fortgeführt (Lindmeier und Heinze 2020). Konkret werden im Modell drei Schichten vorgeschlagen, die sich in ihrer Funktion unterscheiden. Die äußerste Schicht stellt Differenzierung und Adaptivität dar. Diese wird als notwendige Grundlage aller anderen Dimensionen und eng verknüpft mit den anderen Dimensionen angesehen. ${ }^{4}$ In der mittleren Schicht finden sich diejenigen Unterrichtsdimensionen - Klassenführung, sozio-emotionale Unterstützung, Unterstützung der aktiven Beteiligung - denen eine unterstützende, aber nicht zwingend notwendige Funktion für die innerste Schicht zugeschrieben wird. Die innerste Schicht schließlich stellen diejenigen Dimensionen dar, die auf die direkte Unterstützung des Lernprozesses der Schüler*innen ausgerichtet sind: Auswahl und Thematisierung von Inhalten und Fachmethoden, kognitive Aktivierung, Unterstützung des Übens und formatives Assessment. Diese Dimensionen hängen aufgrund ihrer Bedeutung für den Lernprozess eng zusammen und sind als tendenzielle, aber nicht strikte Abfolge gedacht.

Der Ausrichtung auf den Lernprozess folgend, wird im Modell die Idee der Angebots-Nutzungs-Modelle (siehe Vieluf et al. 2020) integriert und ein direkter Zusammenhang zwischen der innersten Schicht der Unterrichtsdimensionen und den dadurch initiierten Lernprozessen auf Seiten der Schüler*innen angenommen; die weiteren Schichten sollten ebenfalls mit den Lernprozessen verbunden sein, aber lediglich indirekt vermittelt über die innerste Schicht. Die Lernprozesse wiederum sollten zur Weiterentwicklung bestimmter Outcomes auf Seiten der Schüler*innen führen. Die Outcomes sind in Anlehnung an das Dynamic Model of Educational Effectiveness unterteilt in kognitive, meta-kognitive, psychomotorische und affektive Outcomes.

Das Modell befindet sich aktuell noch in Entwicklung und ist empirisch bislang nicht überprüft. Einige Elemente des Modells bedürfen daher der weiteren Ausdifferenzierung und Überarbeitung. So wird beispielsweise im Geschichts-Beitrag auf die enge Verwobenheit der Dimensionen sozio-emotionale Unterstützung und kognitive Aktivierung für bestimmte geschichtliche Unterrichtsinhalte hingewiesen. Auch andere Dimensionen, wie beispielsweise die Unterstützung des Übens und kognitive Aktivierung, weisen Überschneidungen auf (siehe Abschnitte 4.1 und 4.2). Solche Abhängigkeiten finden sich aktuell im Modell nicht. Zudem kann kritisch hinterfragt werden, inwiefern die aus Gründen der Komplexitätsreduktion gewählte unidirektionale Wirkungsdarstellung vom Unterrichtsangebot zur Nutzung desselben aufgrund der Ko-Konstruktion von Unterricht irreführend ist. Schließlich wird in Bezug auf die Lernprozesse bislang keine Unterscheidung verschiedener Dimensionen vorge-

\footnotetext{
${ }^{4}$ Die im Syntheseframework von Praetorius und Charalambous (2018) enthaltene Dimension Unterstützung des Lernens aller Schüler*innen wurde von Charalambous und Praetorius (2020) in zwei Dimensionen separiert: Differenzierung und Adaptivität (äußerste Schicht) und Unterstützung der aktiven Beteiligung (mittlere Schicht).
} 
nommen und diese auch nicht auf spezifische Unterrichtsdimensionen bezogen, da dies basierend auf dem aktuellen Forschungsstand nicht sinnvoll möglich erschien (Charalambous und Praetorius 2020). Dies stellt einen wichtigen nächsten Schritt in der empirischen Überprüfung und theoretischen Weiterentwicklung des Modells dar. Auch für diese Weiterentwicklung ist die enge Zusammenarbeit und der Austausch zwischen allgemeiner und fachspezifischer Unterrichtsforschung aus unserer Sicht unabdingbar, um die unterschiedlichen Expertisen zielführend zusammenzubringen.

\subsection{Der praktische Nutzen: Die Verbesserung von Unterricht}

Ein letztes Ziel des erweiterten Syntheseframeworks stand nicht im Vordergrund des Themenheftes, soll hier aber abschließend skizziert werden: Die Suche nach dem Gral ist letztlich dadurch motiviert, Hilfestellung zur Gestaltung von Unterricht zu erhalten - in unserem Fall: ein Instrument zu entwickeln, das sich als Analysegrundlage für die Verbesserung von Unterricht eignet. Die Dimensionen des Modells dienen hier gleichsam als unterschiedliche Perspektiven, die man für die Analyse von Unterricht verwendet und die je nach Dimension bestimmte Unterrichtsaspekte mehr in den Vorder- oder den Hintergrund treten lassen. Wie lässt - oder ließe sich das erweiterte Syntheseframework in der Praxis anwenden?

Eine erste Anwendung besteht in der Analyse von Unterricht, z. B. im Fall von Unterrichtsbeobachtungen im Rahmen der Schulinspektion (siehe Rogh et al. 2020). Für die Bewertung der Unterrichtsqualität durch Beobachter*innen haben Dreher und Leuders einen interessanten Vorschlag unterbreitet: Statt zwischen allgemeinen und fachspezifischen Dimensionen der Unterrichtsqualität zu unterscheiden, könnte man stärker die fachspezifische Expertise der Beobachtenden sicherstellen, um so die Fachspezifität in allgemein formulierten (Sub-)Dimensionen ergänzen zu können. Dieser Gedanke lässt sich weiterführen: Für die Beurteilung von Unterricht ebenso wie die für die Operationalisierung der Dimensionen kann die Zusammenarbeit von Personen unterschiedlicher Perspektive - beispielsweise von Praktiker*innen und Wissenschaftler*innen - unterstützend sein. In der Lehr-Lernforschung werden derartige Formen der engen Zusammenarbeit von Wissenschaft und Lehrpraxis seit vielen Jahren untersucht (z. B. Grosche et al. 2020; Gräsel und Parchmann 2004). Insgesamt zeigt sich in Arbeiten der Unterrichtsqualität und Unterrichtsentwicklung, dass Multi-Perspektivität und die entsprechende Kombination unterschiedlicher Expertise dazu führen, dass Dimensionen konkretisiert und kontextualisiert werden und dabei auch die Grenzen der Anwendbarkeit beleuchtet werden. Dadurch werden sie anschlussfähiger an Diskurse in Wissenschaft und Praxis und können zu einer Weiterentwicklung der theoretischen Überlegungen führen (vgl. Fries et al. 2020).

Eine weitere wichtige Anwendung des Modells ist die Lehrer*innenbildung: Angehende Lehrpersonen müssen lernen, eigenen und fremden Unterricht in Hinblick auf wichtige Qualitätsdimensionen zu beurteilen. Für diese Professionalisierung könnten wichtige Standardsituationen für Dimensionen der Unterrichtsqualität in verschiedenen Fächern bzw. Inhalten auf Video entwickelt werden (vgl. Seidel et al. 2021\#). Anhand dieser Standardsituationen können für die angehenden Lehrpersonen Aufgaben entwickelt werden, mit denen gelernt werden kann, wie Standardsituationen unter Rückgriff auf Expertise bewältigt werden können. 


\section{Fazit und Ausblick}

Welche Erkenntnisse wurden durch diesen erneuten Streifzug der Gralsritter*innen gewonnen? Aus unserer Sicht zeigen beide Themenhefte, dass es sinnvoll ist, einen gemeinsamen Bezugspunkt für den Diskurs zwischen Fachdidaktiken sowie zwischen Fachdidaktiken und der allgemeinen Unterrichtsqualitätsforschung zu nutzen, um so eine gemeinsame Sprache und Struktur für die entsprechenden Diskussionen zugrunde zu legen. In Bezug auf das von uns für diesen Zweck verwendete Syntheseframework zeichnet sich für viele Dimensionen bzw. Subdimensionen ab, dass es einen „Kern“ gibt, auf dessen Relevanz man sich über die verschiedenen Fächer hinweg einigen kann. Der fächerübergreifende Diskurs zum erweiterten Syntheseframework führt darüber hinaus zu wichtigen und innovativen Ergebnissen: Die Erweiterung von kognitiver Aktivierung zu kognitiv-ästhetischer oder kommunikativkognitiver Aktivierung sind dafür wichtige Beispiele. Ein nächster vielversprechender Schritt wäre nun aus unserer Sicht, die erarbeiteten Forschungsstände in konkrete unterrichtliche Standardsituationen zu übertragen (siehe Seidel et al. 2021\#) und diese dann wiederum über Fächer hinweg - und dies idealerweise in einem interdisziplinären, diskursiv angelegten Setting - in den Blick zu nehmen.

Funding Open Access funding enabled and organized by Projekt DEAL.

Open Access Dieser Artikel wird unter der Creative Commons Namensnennung 4.0 International Lizenz veröffentlicht, welche die Nutzung, Vervielfältigung, Bearbeitung, Verbreitung und Wiedergabe in jeglichem Medium und Format erlaubt, sofern Sie den/die ursprünglichen Autor(en) und die Quelle ordnungsgemäß nennen, einen Link zur Creative Commons Lizenz beifügen und angeben, ob Änderungen vorgenommen wurden.

Die in diesem Artikel enthaltenen Bilder und sonstiges Drittmaterial unterliegen ebenfalls der genannten Creative Commons Lizenz, sofern sich aus der Abbildungslegende nichts anderes ergibt. Sofern das betreffende Material nicht unter der genannten Creative Commons Lizenz steht und die betreffende Handlung nicht nach gesetzlichen Vorschriften erlaubt ist, ist für die oben aufgeführten Weiterverwendungen des Materials die Einwilligung des jeweiligen Rechteinhabers einzuholen.

Weitere Details zur Lizenz entnehmen Sie bitte der Lizenzinformation auf http://creativecommons.org/ licenses/by/4.0/deed.de.

\section{Literatur}

Brophy, J. (1986). Teacher influences on student achievement. American Psychologist, 41(10), 1069.

Charalambous, C. Y., \& Praetorius, A. K. (2020). Creating a forum for researching teaching and its quality more synergistically. Studies in Educational Evaluation, 67, 100894. https://doi.org/10.1016/j. stueduc.2020.100894.

Chi, M. T., \& Wylie, R. (2014). The ICAP framework: linking cognitive engagement to active learning outcomes. Educational Psychologist, 49(4), 219-243.

Creemers, B.P. M., \& Kyriakides, L. (2008). The dynamics of educational effectiveness: a contribution to policy, practice, and theory in contemporary schools. London, New York: Routledge.

Decristan, J., Hess, M., Holzberger, D., \& Praetorius, A. K. (2020). Oberflächen-und Tiefenmerkmale: eine Reflexion zweier prominenter Begriffe der Unterrichtsforschung. Zeitschrift für Pädagogik, 66(Beiheft 1), 102-116.

Doyle, W. (1977). Paradigms for research on teacher effectiveness. Review of Research in Education, 5(1), 163-198. 
Dreher, A., \& Leuders, T. (2021). Fachspezifität von Unterrichtsqualität - aus der Perspektive der Mathematikdidaktik. Unterrichtswissenschaft. https://doi.org/10.1007/s42010-021-00116-9.

Eberle, F., Gehrer, K., Jaggi, B., Kottonau, J., Oepke, M., \& Pflüger, M. (2008). Evaluation der Maturitätsreform 1995 (EVAMAR). Schlussbericht zur Phase II. Bern: Staatssekretariat für Bildung und Forschung SBF.

Ericsson, K. A. (1996). The acquisition of expert performance: an introduction to some of the issues. In K. A. Ericsson (Hrsg.), The road to excellence: the acquisition of expert performance in the arts and sciences, sports, and games (S. 1-50). Mahwah: Erlbaum.

Ericsson, K. A., \& Harwell, K. W. (2019). Deliberate Practices and proposed limits on the effects of practice on the acquisition of expert performance: Why the original definition matters and recommendations for future research. Frontiers in Psychology. https://doi.org/10.3389/fpsyg.2019.02396.

Fries, L., Son, J. Y., Givvin, K. B., \& Stigler, J. W. (2020). Practicing connections: a framework to guide instructional design for developing understanding in complex domains. Educational Psychology Review, 33, 739-762.

Gage, N.L., \& Needels, M.C. (1989). Process-product research on teaching: a review of criticisms. The elementary school journal, 89(3), 253-300.

Gräsel, C., \& Parchmann, I. (2004). Implementationsforschung - oder: der steinige Weg, Unterricht zu verändern. Unterrichtswissenschaft, 32(3), 196-214.

Gräsel, C., Decristan, J., \& König, J. (2017). Adaptiver Umgang mit Heterogenität im Unterricht. Unterrichtswissenschaft, 45, 195-206.

Gräsel, C., Neumann, K., Gruber, H., Rothgangel, M., \& Prenzel, M. (2021). Der Einfluss von Fritz Oser auf vier Themen der Unterrichts- und Schulforschung: eine dankbare Rückschau und ein Blick in die Zukunft. Unterrichtswissenschaft, 49(1), 1-16.

Grosche, M., Fussangel, K., \& Gräsel, C. (2020). Kokonstruktive Kooperation zwischen Lehrkräften: Aktualisierung und Erweiterung der Kokonstruktionstheorie sowie deren Anwendung am Beispiel schulischer Inklusion. Zeitschrift für Pädagogik, 66(4), 461-479.

Gruehn, S. (2000). Unterricht und schulisches Lernen. Schüler als Quellen der Unterrichtsbeschreibung. Münster: Waxmann.

Hellermann, B., Reinhardt, V., Rehm, M., \& Wilhelm, M. (2021). Ein metaanalytischer Überblick auf der Basis fachunterrichtlicher Expertenzusammenfassungen zu wirksamem Unterricht in siebzehn Fächern. In V. Reinhardt, M. Rehm \& M. Wilhelm (Hrsg.), Wirksamer Fachunterricht - Metaband. Eine metaanalytische Betrachtung von Expertisen aus 17 Schulfächern (S. 309-366). Baltmannsweiler: Schneider.

Helmke, A. (2017). Unterrichtsqualität und Lehrerprofessionalität. Diagnose, Evaluation und Verbesserung des Unterrichts (7. Aufl.). Seelze: Klett.

Hermann, C., \& Gerlach, E. (2020). Unterrichtsqualität im Fach Sport - Ein Überblick zum Forschungsstand in Theorie und Empirie. Unterrichtswissenschaft, 48(3), 361-384.

Hesse, F. (2021). Unterrichten üben im Schulpraktikum. Theoretische Überlegungen und Konzeption einer Videostudie zum Literaturunterricht von Studierenden. In J. Heins, K. Kleinschmidt-Schinke, D. Wieser \& E. Wiesner (Hrsg.), Üben. Theoretische und empirische Perspektiven in der Deutschdidaktik. SLLD-B.

Hiebert, J., \& Stigler, J. W. (in press). Creating practical theories of teaching. In A.-K. Praetorius \& C. Y. Charalambous (Hrsg.), Theorizing teaching: Bringing together expert perspectives to move the field forward. Berlin Heidelberg: Springer.

Hugener, I. (2008). Inszenierungsmuster im Unterricht und Lernqualität. Münster: Waxmann.

Kirchner, C., \& Kirschenmann, J. (2015). Kunst unterrichten. Didaktische Grundlagen und schülerorientierte Vermittlung. Hamburg: Seelze.

Kirschner, P. A., Verschaffel, L., Star, J., \& Van Dooren, W. (2017). There is more variation within than across domains: an interview with Paul A. Kirschner about applying cognitive psychology-based instructional design principles in mathematics teaching and learning. ZDM Mathmatics Education, 49(4), 637-643.

Klauer, K.J., \& Leutner, D. (2012). Lehren und Lernen. Einführung in die Instruktionspsychologie (2. Aufl.). Weinheim: Beltz.

Klieme, E. (2019). Unterrichtsqualität. In M. Gläser-Zikuda, M. Harring \& C. Rohlfs (Hrsg.), Handbuch Schulpädagogik (S. 393-408). Münster: Waxmann.

Klieme, E., \& Rakoczy, K. (2008). Empirische Unterrichtsforschung und Fachdidaktik. Outcome-orientierte Messung und Prozessqualität des Unterrichts. Zeitschrift für Pädagogik, 54(2), 222-237.

Klieme, E., \& Warwas, J. (2011). Konzepte der individuellen Forderung. Zeitschrift für Pädagogik, 57, 805-818. 
Klieme, E., Pauli, C., \& Reusser, K. (2009). The Pythagoras study: investigating effects of teaching and learning in Swiss and German mathematics classrooms. In T. Janik \& T. Seidel (Hrsg.), The power of video studies in investigating teaching and learning in the classroom (S. 137-160). Münster: Waxmann.

Klieme, E., Schümer, G., \& Knoll, S. (2001). Mathematikunterricht in der Sekundarstufe I. In Bundesministerium für Bildung und Forschung (Hrsg.), TIMSS - Impulse für Schule und Unterricht. Forschungsbefunde, Reforminitiativen, Praxisberichte und Videodokumente (S. 42-57). Bonn: Bundesministerium für Bildung und Forschung.

Kranefeld, U. (2021). Der Diskurs um Unterrichtsqualität in der Musikdidaktik zwischen generischen und fachspezifischen Dimensionen. Unterrichtswissenschaft. https://doi.org/10.1007/s42010-021-00113$\mathrm{y}$.

Kunter, M., \& Ewald, S. (2016). Bedingungen und Effekte von Unterricht: Aktuelle Forschungsperspektiven aus der pädagogischen Psychologie. In N. McElvany, W. Bos, H. G. Holtappels, M. M. Gebauer \& F. Schwabe (Hrsg.), Bedingungen und Effekte guten Unterrichts (S. 9-31). Münster: Waxmann.

Lindmeier, A., \& Heinze, A. (2020). Die fachdidaktische Perspektive in der Unterrichtsqualitätsforschung: (bisher) ignoriert, implizit enthalten oder nicht relevant? Zeitschrift für Pädagogik, 66(Beiheft 1), $255-268$.

Macnamara, B. N., Hambrick, D.Z., \& Oswald, F. L. (2014). Deliberate practice and performance in music, games, sports, education, and professions: a meta-analysis. Psychological Science, 25, 1608-1618.

Martin-Raugh, M., Tannenbaum, R.J., Tocci, C. M., \& Reese, C. (2016). Behaviorally anchored rating scales: an application for evaluating teaching practice. Teaching and Teacher Education, 59, 414-419.

Neuhaus, B.J. (2021). Unterrichtsqualität aus der Perspektive der Biologiedidaktik. Unterrichtswissenschaft. https://doi.org/10.1007/s42010-021-00114-x.

Neumann, K. (2018). Unterrichtsqualität in den Naturwissenschaften - Die Suche nach dem Heiligen Gral. In C. Maurer (Hrsg.), Qualitätsvoller Chemie- und Physikunterricht - normative und empirische Dimensionen. Gesellschaft für Didaktik der Chemie und Physik Jahrestagung. Regensburg, 2017. (S. 5-18).

Openshaw, K., \& Clarke, S.C.T. (1970). General teaching theory. Journal of Teacher Education, 21(3), 403-416.

Oser, F., \& Baeriswyl, F. J. (2001). Choreographies of teaching: bridging instruction to learning. In V. Richardson (Hrsg.), Handbook of research on teaching (S. 1031-1065). Washington, DC: American Educational Research Association.

Pianta, R.C., Hamre, B. K., \& Mintz, S. (2012). Classroom assessment scoring system upper elementary manual. Charlottesville: Teachstone.

Praetorius, A.-K., \& Charalambous, C. Y. (2018). Classroom observation frameworks for studying teaching quality: looking back and looking forward. ZDM Mathematics Education, 50(3), 535-553.

Praetorius, A.-K., Klieme, E., Herbert, B., \& Pinger, P. (2018). Generic dimensions of teaching quality: the German framework of three basic dimensions. ZDM, 50(3), 407-426.

Praetorius, A.-K., Herrmann, C., Gerlach, E., Zülsdorf-Kersting, M., Heinitz, B., \& Nehring, A. (2020a). Unterrichtsqualität in den Fachdidaktiken im deutschsprachigen Raum - zwischen Generik und Fachspezifität. Unterrichtswissenschaft, 48(3), 409-446.

Praetorius, A.-K., Klieme, E., Kleickmann, T., Brunner, E., Lindmeier, A., Taut, S., \& Charalambous, C. (2020b). Towards developing a theory of generic teaching quality. Origin, current status, and necessary next steps regarding the Three Basic Dimensions Model. Zeitschrift für Pädagogik, 66(Beiheft 1/20), 15-36.

Rakoczy, K., Wagner, E., \& Frick, U. (2021). Wie in Mathe so auch in Kunst? Zur Konzeption von Unterrichtsqualität im Kunstunterricht. Unterrichtswissenschaft. https://doi.org/10.1007/s42010-02100104-z.

Renkl, A. (2012). Modellierung von Kompetenzen oder von interindividuellen Kompetenzunterschieden. Ein unterschätzter Unterschied. Psychologische Rundschau, 63(1), 50-53.

Renkl, A. (2017). Instruction based on examples. In R. E. Mayer \& P. A. Alexander (Hrsg.), Handbook of research on learning and instruction (2. Aufl. S. 325-348). New York: Routledge.

Reusser, K., \& Pauli, C. (2021). Unterrichtsqualität ist immer generisch und fachspezifisch. Ein Kommentar aus kognitions- und lehr-lerntheoretischer Sicht. Unterrichtswissenschaft. https://doi.org/10.1007/ s42010-021-00117-8.

Richartz, A., \& Kohake, K. (2021). Zur (Fach-)Spezifität von Unterrichtsqualität im Fach Sport. Unterrichtswissenschaft. https://doi.org/10.1007/s42010-021-00112-z. 
Rogh, W., Praetorius, A.-K., Gossner, L., \& Wehrli, F. (2020). Unveröffentlichtes Instrument zur Unterrichtsbeurteilung im Rahmen der externen Schulevaluation in der deutschsprachigen Schweiz. Zürich, Schweiz: Universität Zürich.

Rothgangel, M., Abraham, U., Bayrhuber, H., Federking, V., Jank, W., \& Vollmer, H. J. (2021). Lernen im Fach und über das Fach hinaus. Bestandsaufnahmen und Forschungspsperspektiven aus 17 Fachdidaktiken im Vergleich. Münster: Waxmann.

Rothgangel, M. (2021). Unterrichtsqualität in der Religionsdidaktik - fachspezifische und fachübergreifende Aspekte. Unterrichtswissenschaft. https://doi.org/10.1007/s42010-021-00107-w.

Scheerens, J. (2015). Theories on educational effectiveness and ineffectiveness. School Effectiveness and School Improvement, 26, 10-31. https://doi.org/10.1080/09243453.2013.858754.

Schilcher, A., Krauss, S., Kirchhoff, P., Lindl, A., Hilbert, S., Asen-Molz, K., Ehras, C., et al. (2021). FALKE: Experiences from transdisciplinary educational research by fourteen disciplines. Frontiers in Education. https://doi.org/10.3389/feduc.2020.579982.

Schmidt, H. G., \& Boshuizen, H.P. (1993). On acquiring expertise in medicine. Educational Psychology Review, 5(3), 205-221.

Schoenfeld, A.H. (2018). Video analyses for research and professional development: the teaching for robust understanding (TRU) framework. ZDM Mathematics Education, 50(3), 491-506.

Schwartz, D., Bransford, J., \& Sears, D. (2005). Efficiency and innovation in transfer. In J. Mestre (Hrsg.), Transfer of learning from a modern multidisciplinary perspective (S. 1-51). Greenwich: Information Age Publishing.

Seidel, T., \& Shavelson, R. J. (2007). Teaching effectiveness research in the past decade: The role of theory and research design in disentangling meta-analysis results. Review of Educational Research, 77(4), 454-499.

Seidel, T., Renkl, A., \& Rieß, W. (2021). Basisdimensionen für Unterrichtsqualität im Fachkontext konkretisieren: Gestaltungsmerkmale und Bestimmung von Standardsituationen. Unterrichtswissenschaft. https://doi.org/10.1007/s42010-021-00108-9.

Trautwein, U., Schreiber, W., \& Hasenbein, L. (2021). Erkennt und nutzt die geschichtsdidaktische Forschung das Potenzial der Basisdimensionen der Unterrichtsqualität? Unterrichtswissenschaft. https:// doi.org/10.1007/s42010-021-00120-z.

Vieluf, S., Praetorius, A.K., Rakoczy, K., Kleinknecht, M., \& Pietsch, M. (2020). Angebots-NutzungsModelle der Wirkweise des Unterrichts: ein kritischer Vergleich verschiedener Modellvarianten. Zeitschrift für Pädagogik, 66(Beiheft 1), 63-80.

Wilden, E. (2021). Fachspezifische Aspekte von Unterrichtsqualität im Schulfach Englisch. Unterrichtswissenschaft. https://doi.org/10.1007/s42010-021-00105-y.

Winne, P.H. (1987). Why process-product research cannot explain process-product findings and a proposed remedy: the cognitive mediational paradigm. Teaching and Teacher Education, 3(4), 333-356.

Wiprächtiger-Geppert, M., Stahns, R., \& Riegler, S. (2021). Fachspezifität von Unterrichtsqualität in der Deutschdidaktik. Unterrichtswissenschaft. https://doi.org/10.1007/s42010-021-00109-8.

Zülsdorf-Kersting, M. (2020). Qualitätsmerkmale von Geschichtsunterricht. Zum Verhältnis generischer und fachspezifischer Merkmale. Unterrichtswissenschaft, 48(3), 385-407. 